\title{
LiDAR with Pulsed Time of Flight
}

${ }^{1}$ S M Nazmuz Sakib (Orchid- https://orcid.org/0000-0001-9310-3014) (sakibpedia@gmail.com)

${ }^{1}$ Graduate of BSc in Business Studies

School of Business And Trade

Pilatusstrasse 6003, 6003 Luzern,

Switzerland
${ }^{1}$ Student of LLB(Hon's)

Department of Law

Dhaka International University

House \# 4, Road \# 1, Block - F, Dhaka 1213

\section{Abstract}

LIDAR data has proved useful in recent years since it gives information such as item height and attributes, statistics over large regions, and it all becomes available by capturing the intensity of backscattered pulses in addition to 3D coordinates. A LiDAR may generate a 2D angle image as well as a 3D picture containing angle/angle/range information. A LiDAR can directly measure range in each pixel since it controls when light is emitted, allowing it to calculate range based on the time of flight (ToF) to and from the object in a particular pixel. If there is enough signal, a 3D picture can also have grayscale and colour . Coherent LiDAR can detect velocity quite precisely. Brief descriptions of airborne laser scanning technology [also known as "light detection and ranging" (LIDAR)] and research findings on its application in forest assessment and monitoring are included. Many airborne laser scanning missions are being flown with terrain mapping requirements, leading to data sets that are missing important data for vegetation evaluation. As a result, standards and criteria for airborne laser scanning missions are needed to guarantee that they are used for vegetation measurement and monitoring instead of merely landscape mapping (e.g., delivery of all return data with reflection intensity).

Keywords: LIDAR, 3D, ToF, Pulsed Time of Flight, EM, Wavelengths, Electromagnetic pulse.

\section{Introduction}

LiDAR employs electromagnetic (EM) pulses of optical and infrared wavelengths. It is an active sensor, which means that it emits an EM pulse and receives the signal that bounces back. It is similar to microwave radar, but with a much shorter wavelength. This means it will have far greater angular resolution than radar but will be unable to see through fog or clouds . It is similar to passive electro-optical (EO) sensors in wavelengths, except that it creates its own radiation 
rather than utilizing existing radiation and has several more sensing modes due to control over the scene illumination (De Raedt et al., 2009, P.1/10).

\subsection{Definition of Key Terms}

\subsubsection{LIDAR}

LIDAR employs lasers to determine the heights of objects such as the ground, woods, and even buildings. It resembles a radar, which uses radio waves to map things, or a sonar, which uses sound waves to map things, but a LIDAR system utilizes light emitted by a laser to map things. LIDAR, also known as LADAR, is an optical remote sensing technique that detects the

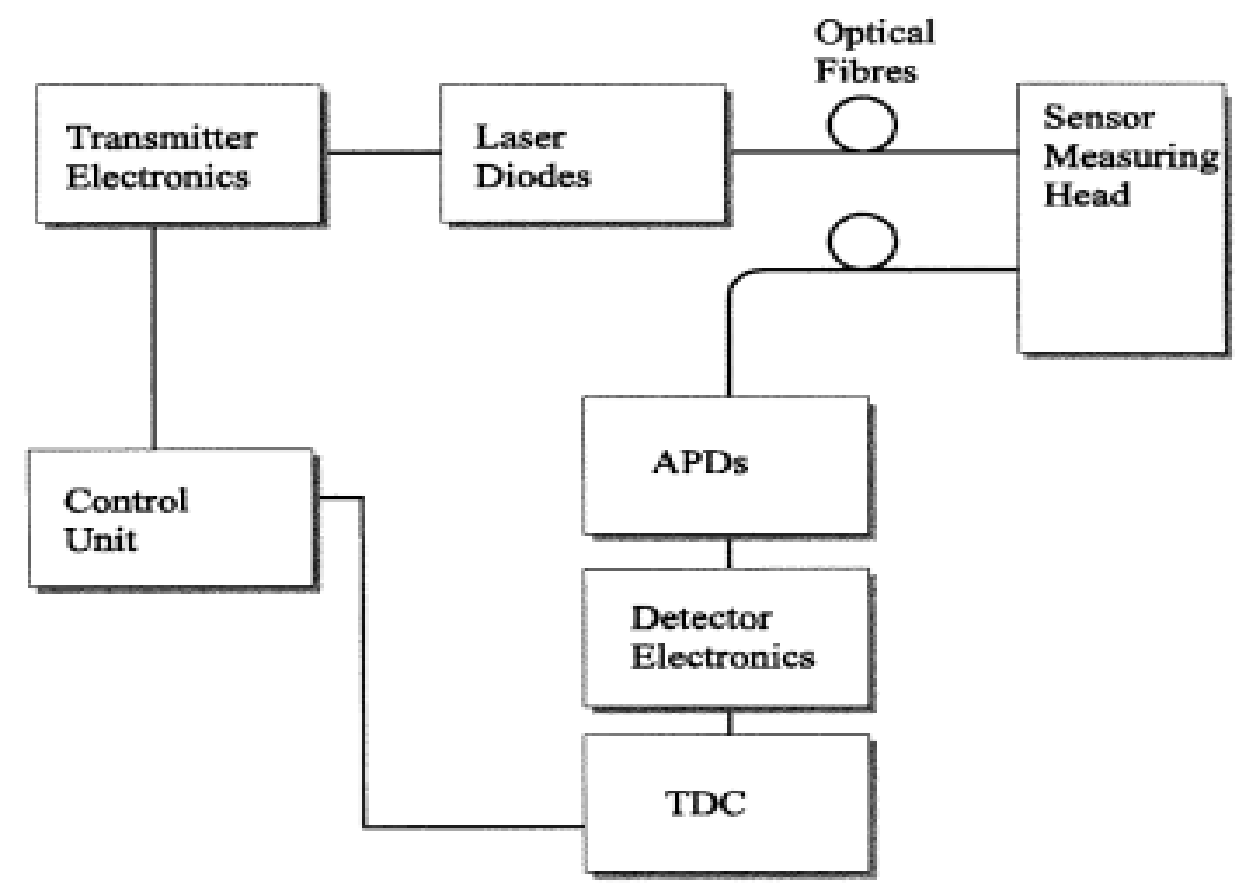

characteristics of scattered light to determine the range and/or other information of a distant target structure (Olsen, 2016, P.9/29).

Figure 1: Overall construction of the LIDAR (Myllylä et al., 1998, P.3/7).

\subsubsection{ToF (Time of Flight)}

Lasers are used in LIDAR to estimate the heights of things like the ground, trees, and even buildings. A LIDAR system is similar to a radar in that it employs radio waves to map things and 
a sonar in that it uses sound waves to map things, but it uses light generated by a laser to map things. LIDAR, also referred as LADAR, is an optical remote sensing technology for considering a range and/or other data of a distant target structure by detecting the properties of scattered light.

ToF systems are divided into two types:

Indirect (I-ToF): In I-ToF systems, depth information is computed by detecting the phase shift of the intercepted pulsed or modulated light with reference to the released one .

D-ToF: D-ToF uses avalanche detectors' excellent temporal precision to directly estimate the time of flight of the outgoing light pulse (Tontini et al., 2020, P.1-2/19)

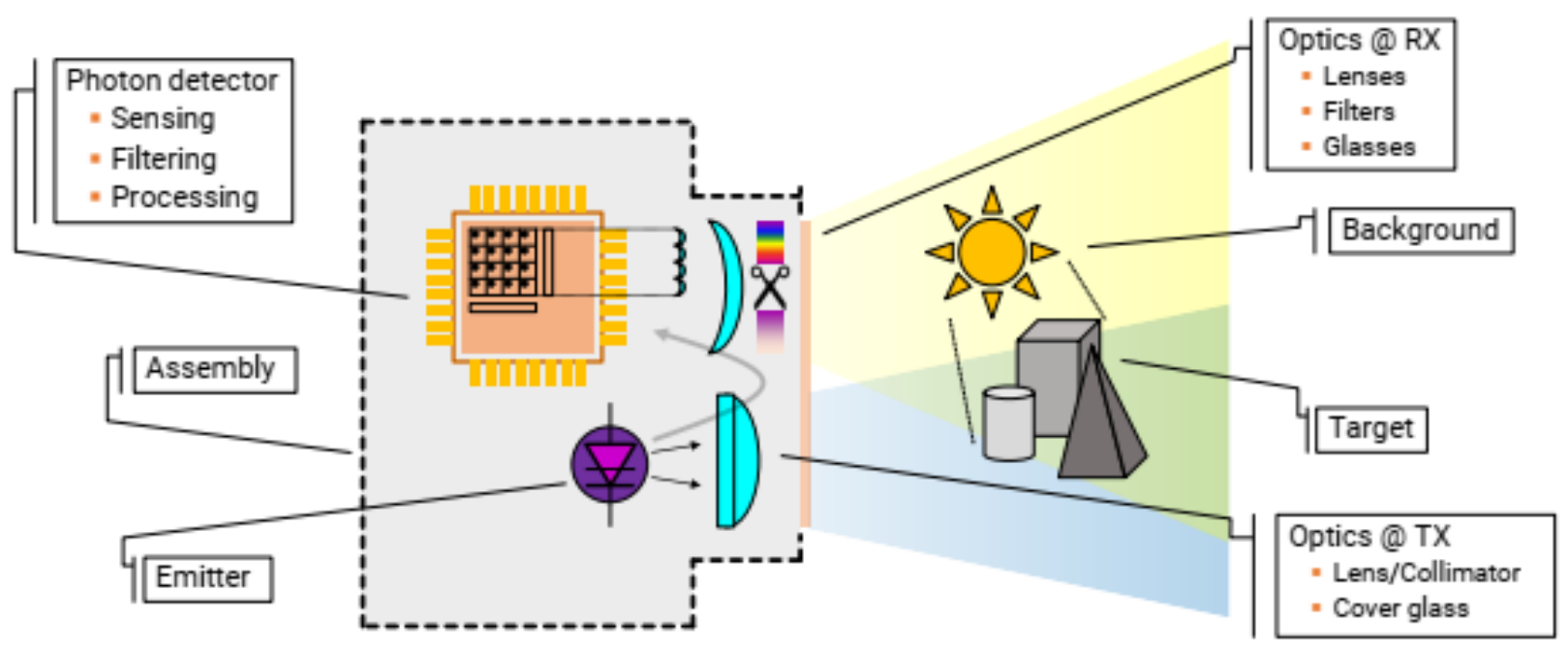


Figure 2: A D-ToF system's block diagram (Tontini et al., 2020, P.2/19)

\section{Time of flight}

\section{Time it takes for emitted pulse to reflect off object and return to scanner.}

$$
\text { Distance }=\frac{\text { Speed of Light } \times \text { Time of Flight }}{2}
$$

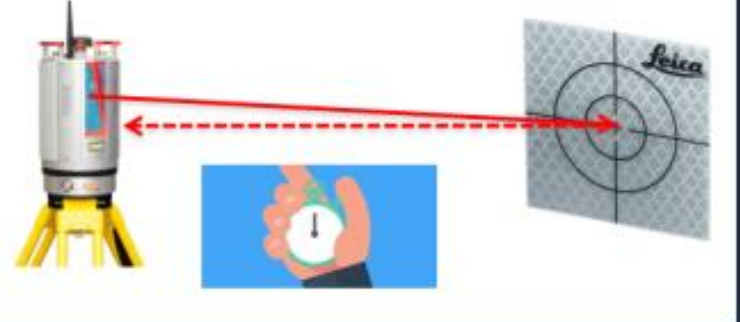

\section{Phase Shift}

Distance is calculated along a sinusoidally modulated laser pulse.

Time of Flight $=\frac{\text { Phase Shift }}{2 \pi x \text { Modulation Frequency }}$

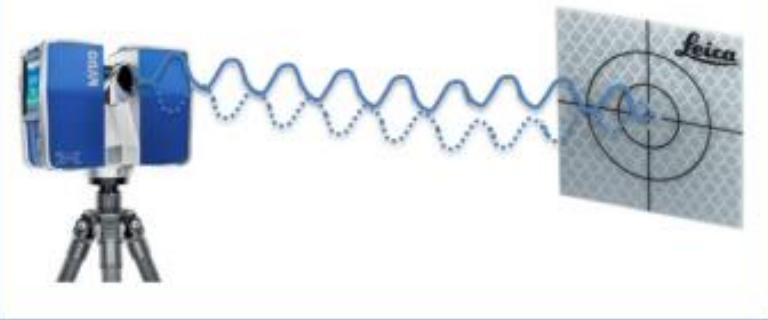

\subsection{Background}

Scanning LiDAR, stereo vision, and structural light sensors are just a few of the sensors that may be utilized to acquire 3D data today. Scanning LiDAR is the most often used sensor for acquiring 3D data due to its accuracy and wide measurement range (Fang et al., 2018, P.1/15). Depending on the power of the transmitter laser, the pulsed time-of-flight method is most commonly employed for measuring distances varying from a few meters to hundreds of meters. Early laser-based distance measuring equipment consisted of manually driven time-of-flight devices intended for multiple-point range measurements. They need precise hand positioning with relation to the thing to be measured, and measurements take time.

Scanner-mirror-based devices comprised the second generation of distance measuring tools. These instruments made use of a variety of scanning methods, which resulted in variable sensor performance in terms of scanning speed and picture resolution (Myllylä et al., 1998, P.2/7). 


\subsection{Concept of the imaging LIDAR}

Imaging LIDAR is a hybrid device that employs a laser array as the transmitter and a detector array as the receiver. Light from a laser diode is guided through an optical fiber, where it travels through a transmitter lens before radiating towards the target surface. Each laser in the array illuminates a portion of the target's overall field of vision. The receiver lens collects a part of the light backscattered from the surface and directs it via an optical fiber to the appropriate detector on the matrix (Myllylä et al., 1998, P.3/7).

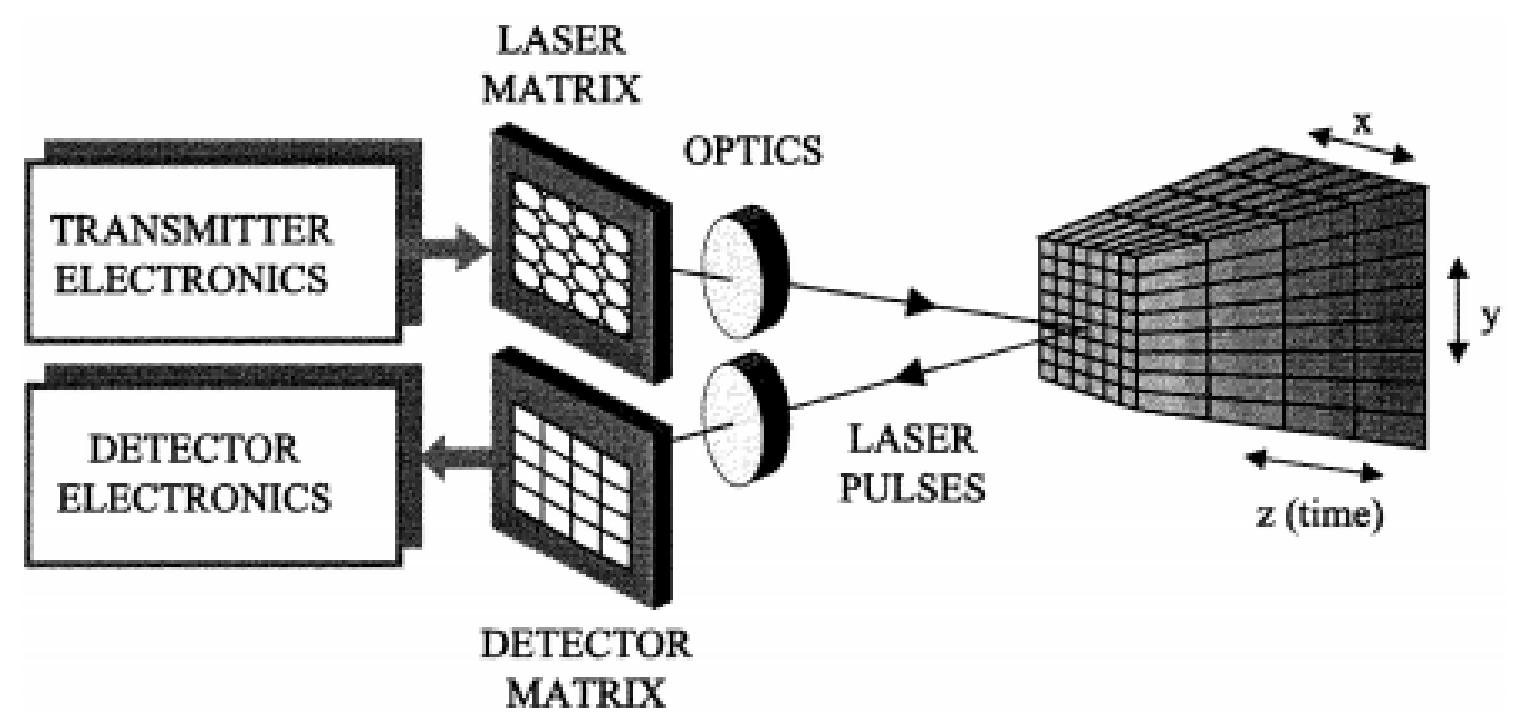

Figure 3: Diagram of the imaging LIDAR (Myllylä et al., 1998, P.3/7).

\subsection{D-laserscanners}

Imaging LIDAR is a hybrid technology that uses a laser array as a transmitter and a detector array as a receiver. Light from a laser diode is steered through an optical fiber, passing via a transmitter lens before propagating towards the target surface. Each laser in the array illuminates a different part of the target's entire field of view. The receiver lens captures some of the light 
backscattered from the surface and sends it to the suitable detector on the matrix through an optical cable (Hildebrandt et al., 2008, P.2/6).

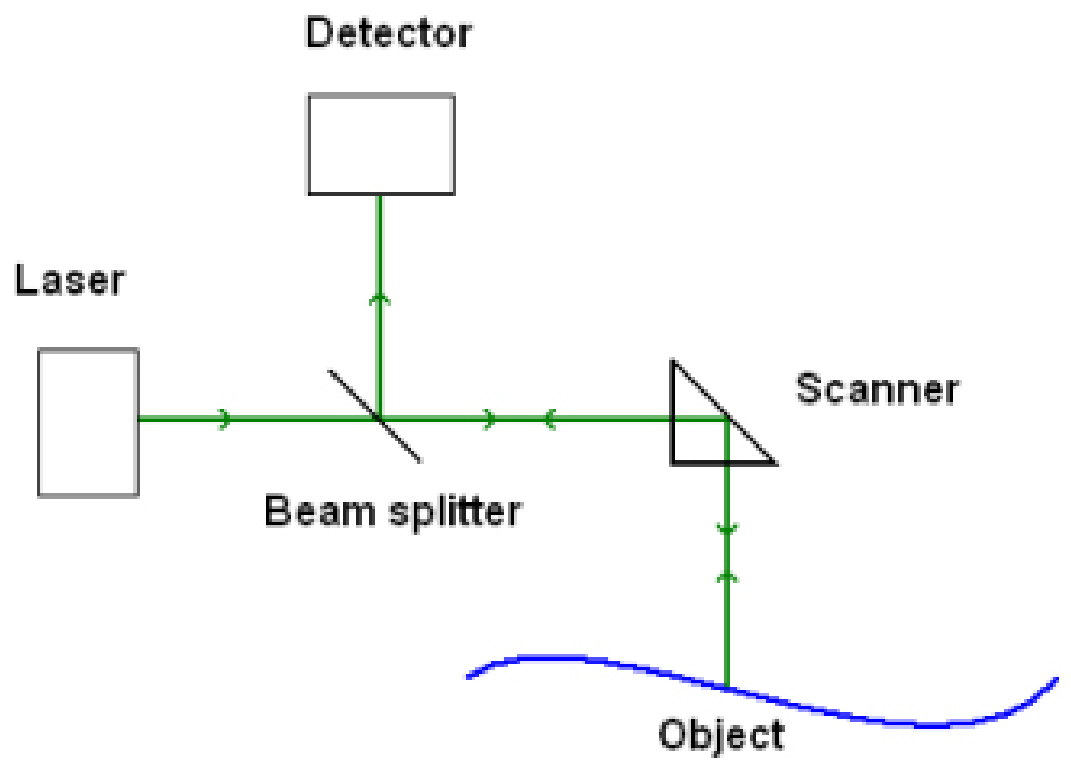

Figure 4: LIDAR-type system using the TOF (time of flight) to obtain depth information (Hildebrandt et al., 2008, P.2/6).

\subsection{Flash LiDAR versus Scanning LiDAR}

\subsubsection{Flash Lidar}

A 3D Flash Lidar emits a pulsed laser beam to the whole solid angle of interest in a single shot. An $n \times m$ array of the photosensitive detector (e.g. arrays of photodiodes or CMOS ToF chip) is essential to get a certain resolution of the point cloud (Maric \& Siedersbeck, 2018. P.4/22). 


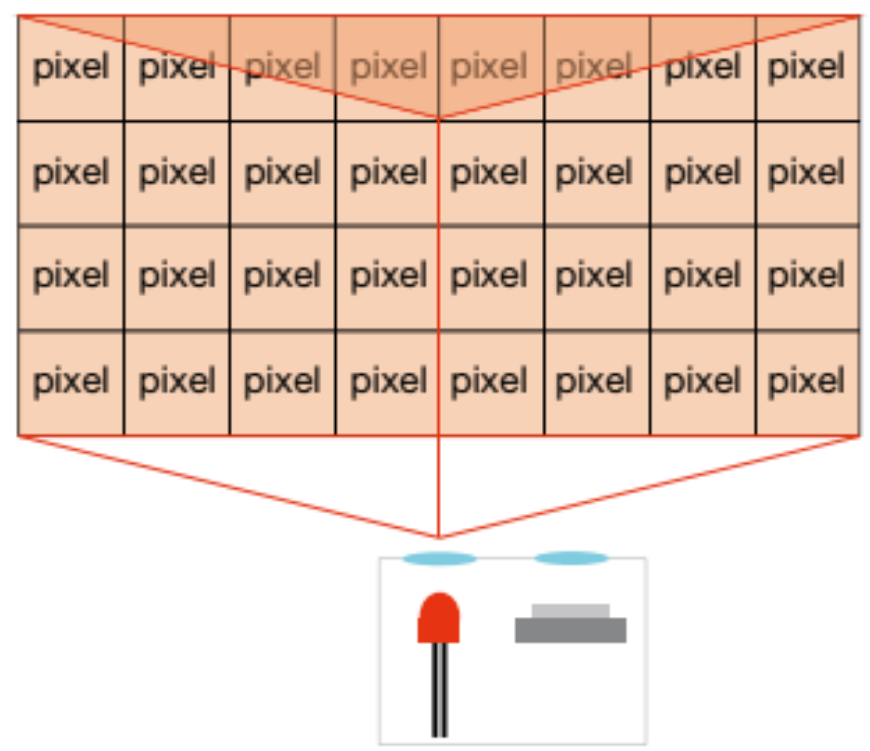

Figure 5: Basic principle of 3D flash Lidar with simultaneous illumination of the target FoV by the laser and its conversion to a certain resolution by an $n x m$ array on the receiver side, e.g. a CMOS ToF camera chip (Maric \& Siedersbeck, 2018, P.5/22).

\section{Flash LiDAR}

-(ㅇ)- Flash LiDAR illuminates the entire scene and uses an array of photodetectors to form an image.
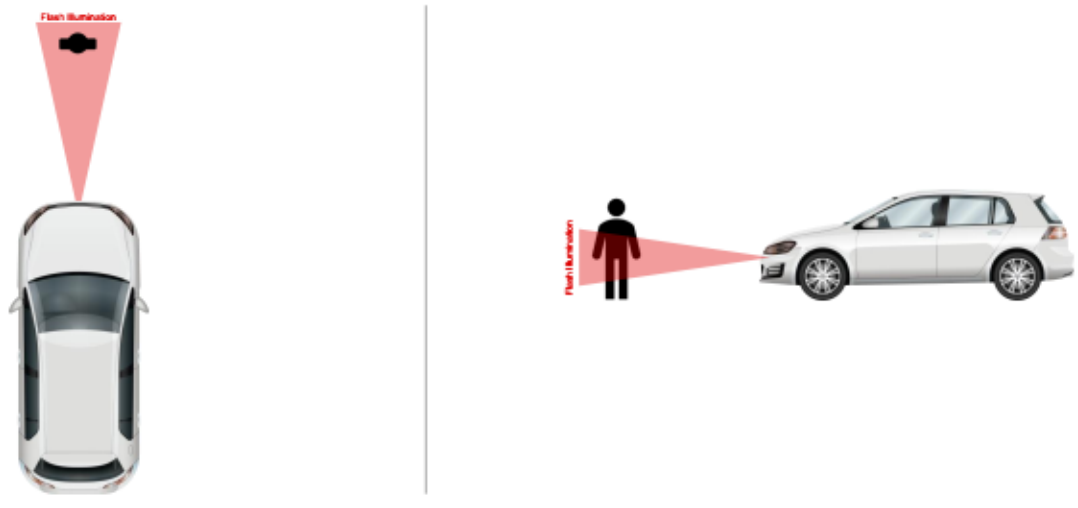

\subsubsection{Scanning LIDAR}

A scanning Lidar system is made up of a focused pulsed laser beam that is directed to a tiny solid angle by either a mechanical rotating mirror or a MEMS (micro electro-mechanical system) mirror. 
Because the high power pulsed laser beam is only projected within a limited solid angle, the attainable distance with the optical power employed may be significantly greater than with 3D Flash systems (Maric \& Siedersbeck, 2018, P.5/22).

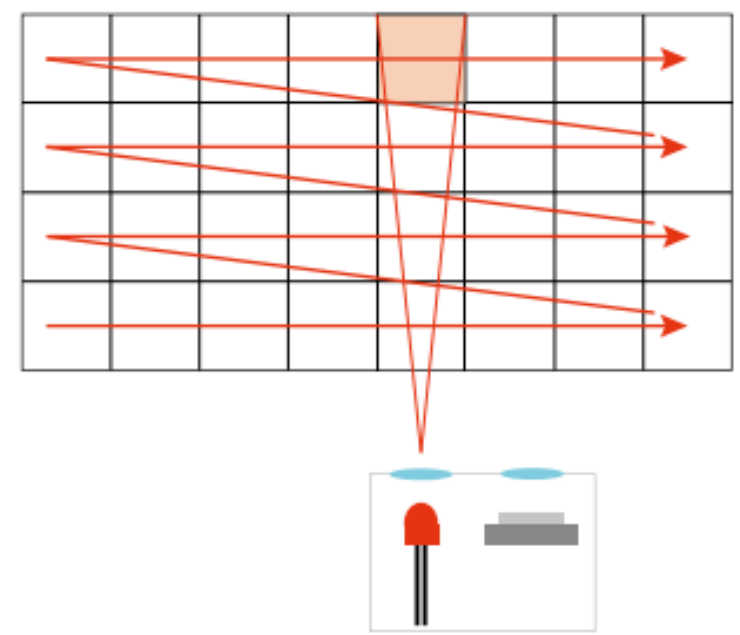

Figure 6: Basic principle of a scanning Lidar system with a focused laser beam directed to a small solid angle and a photodiode that receives the signal from this direction (Maric \& Siedersbeck, 2018,

\section{s c a nning LiDAR}

Most multi-channel scanning LiDAR systems need to scan either horizontally, vertically or both.
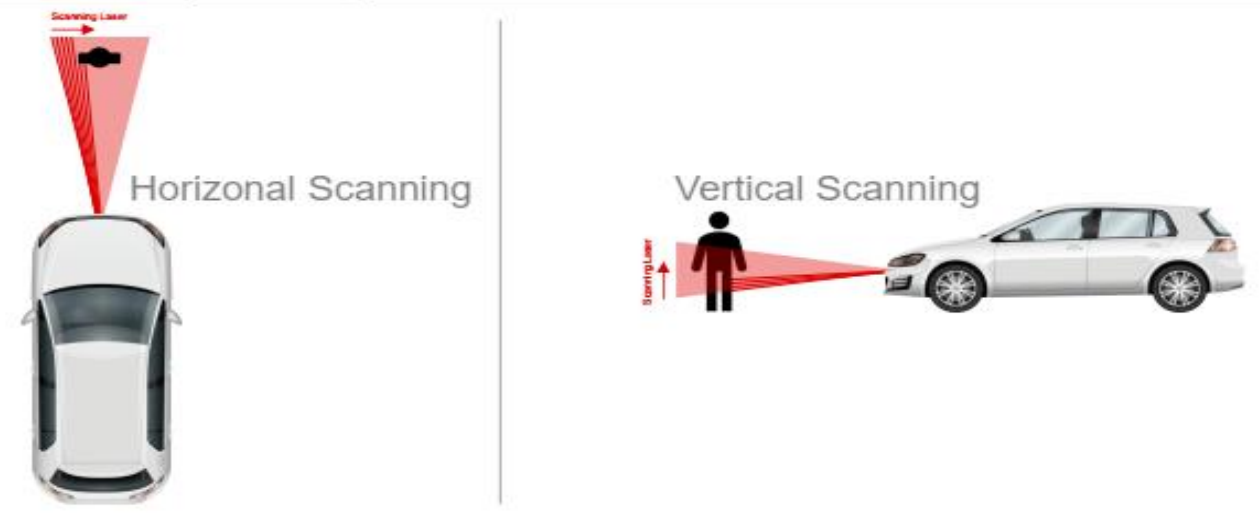

P.5/22). 


\section{Data Analysis}

\subsection{ToF Range Measurement Principle}

ToF technology calculates range by measuring the period it requires for transmitted light to travel to an object and then rebound to the sensor unit. Because of the vacuum speed of light,

$c_{0}=299.792106 \mathrm{~ms}$, is constant (and almost the same as in air), the distance $\mathrm{d}$ is proportional to half the transit time DT, yielding :

$$
d=\frac{1}{2} c_{0} \Delta T
$$

This demands the sensor and the lighting unit being in the same physical location, which is scientifically impossible. The sensor and the lighting unit are placed as near together as possible (Sarbolandi et al., 2018, P.2/22).

ToF allows for the simultaneous recording of the entire image. There is no need for line-by-line scanning or relative motion between the sensor and the objects to be observed. ToF is commonly referred to as LIDAR (light detection and ranging), however this is focused on flash LIDAR rather than scanning LIDAR. ToF may calculate the time of flight of a light pulse in two ways: pulsed operation, which uses charge coupled device (CCD) technology, and continuous wave $(\mathrm{CW})$.

The duration between the emission and reception of a light pulse is determined in pulsed mode, while the phase shift between the emitted and received modulated light pulses is determined in CW mode Because this technology usually relies on high-intensity light pulses transmitted in very brief bursts during a short integration window, pulsed mode is far more resistant to ambient light and therefore more suitable for exterior applications. CW mode, on the other hand, may be easier to create since the light source does not have to be exceedingly short, with quick rising/falling edges (Brand et al., 2019, P.1/2).When contrasted to the phase shift approach, the time-of-flight approach allows for a straightforward system setup and distance computation, but it has a comparatively big measurement error (Brand et al., 2019, P.1/2). 


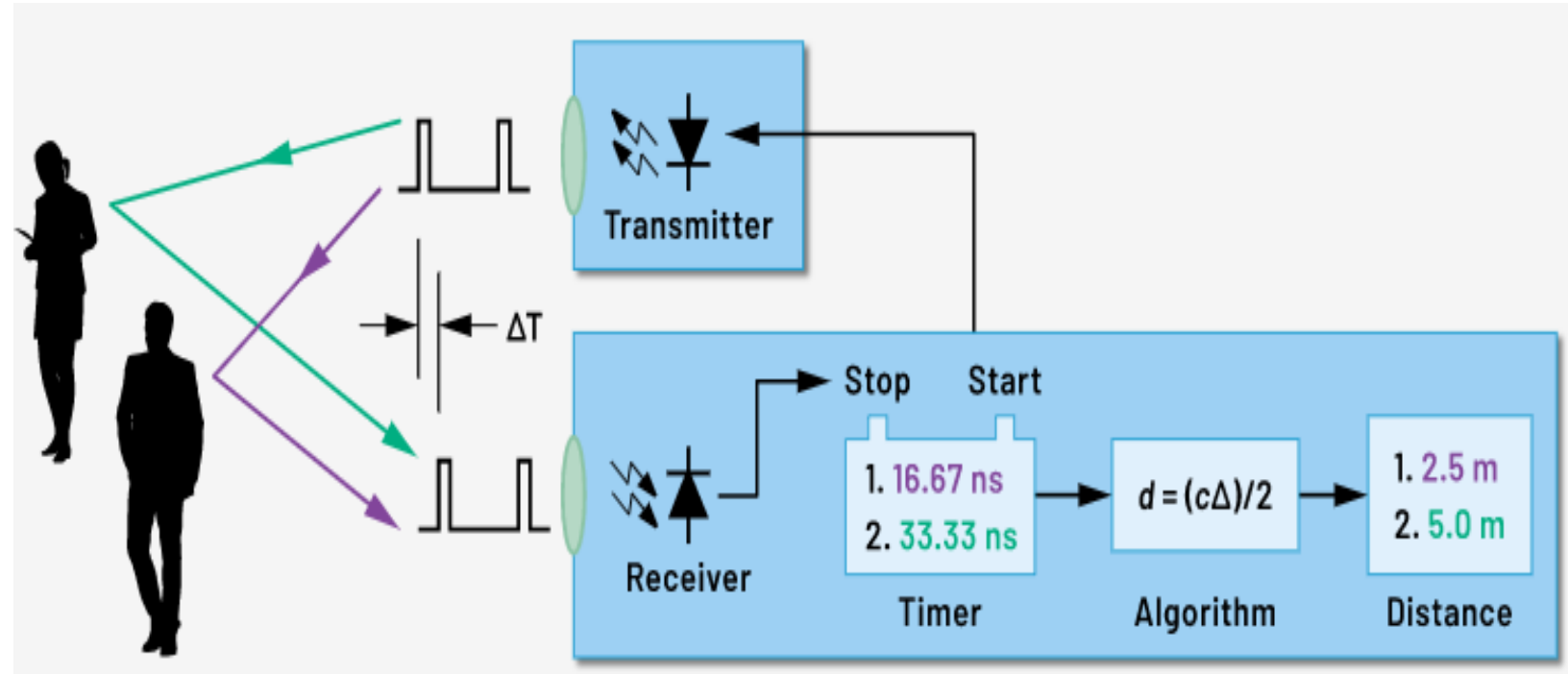

Figure 7: Simple diagram of time of flight measurement (analog.com, 2021).

\subsubsection{Amplitude Modulated Continues-Wave ToF (AMCW-ToF)}

For ToF cameras, the AMCW technique is the most commonly used. The fundamental idea behind the operation is to actively brighten the scene with controlled near infrared (NIR) light. The distance between the sensor and the viewing scene point generates a time shift in the reflected optical signal, which corresponds to a phase shift $\mathrm{f}$ [rad] in a periodic signal with a modulation frequency fm (Sarbolandi et al., 2018, P.2/22).

\subsubsection{Pulse-Based ToF (PB-ToF)}

In principle, a PB-ToF camera generates a single light pulse that is reflected in the scene and gathered by a sensor array. One or more optical or electronic shutters detect the incident light pulse in one or two extremely short temporal windows called gates on the sensor. The amount of incident active light is equivalent to the object distance if the observed object distance is proportionate to the time-of-flight measured in a certain gate (Sarbolandi et al., 2018, P.3/22). 


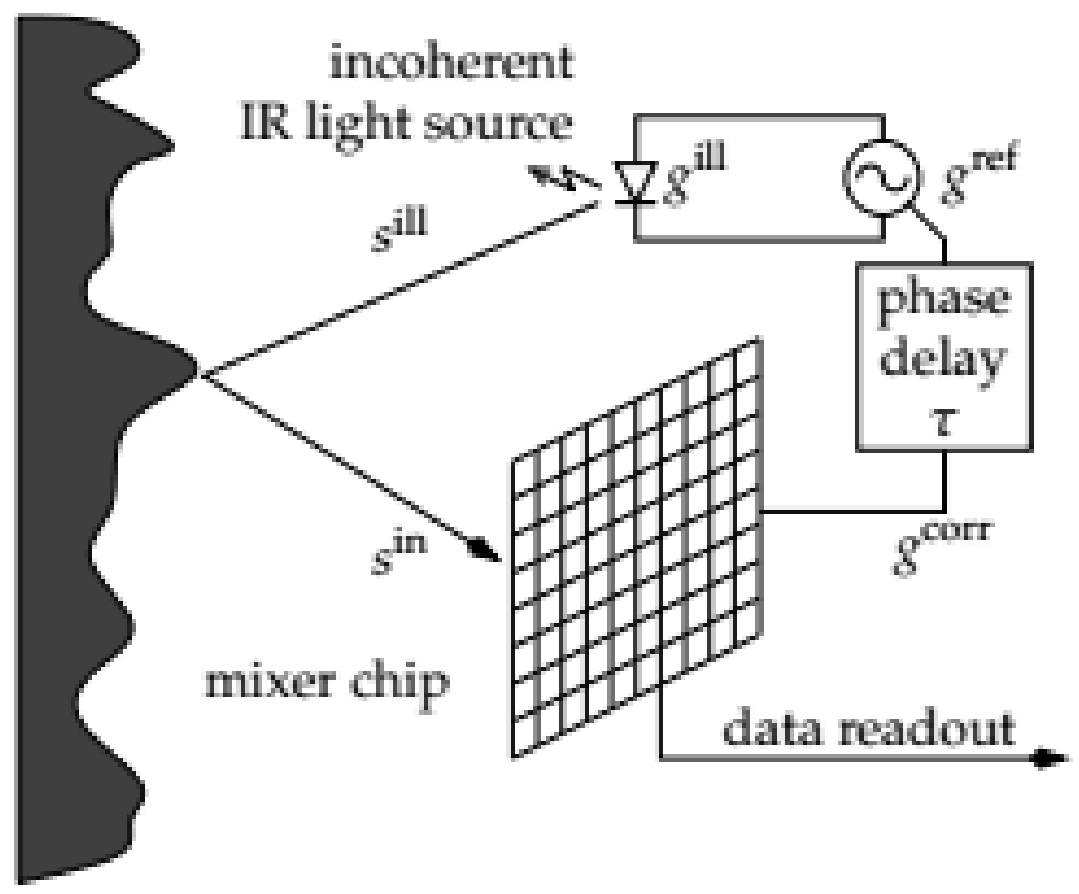

Figure 8: The time of flight (ToF) phase-measurement principle (Sarbolandi et al., 2018, P.2/22).

\subsection{How the object you are detecting affects your ToF measurements}

The photons will always strike something with its particular characteristics, and all surfaces will reflect light uniquely. When light reaches a surface, it can be absorbed, scattered, or transmitted. The percentage of light reflected by a surface is known as its reflectance. Reflectance, on the other hand, is influenced not only by the surface, but also by the type of light reflected. Be cautious: a surface's reflectance for visible and near-infrared light is not quite the same!

When a surface reflects light, it can do so in a variety of ways. Irregular surfaces can make ToF measurements more difficult by making it more difficult for the sensor to discern which photons came from where. Photons can bounce off walls and other objects in confined spaces, significantly complicating the measured distance (terabee, 2021). 


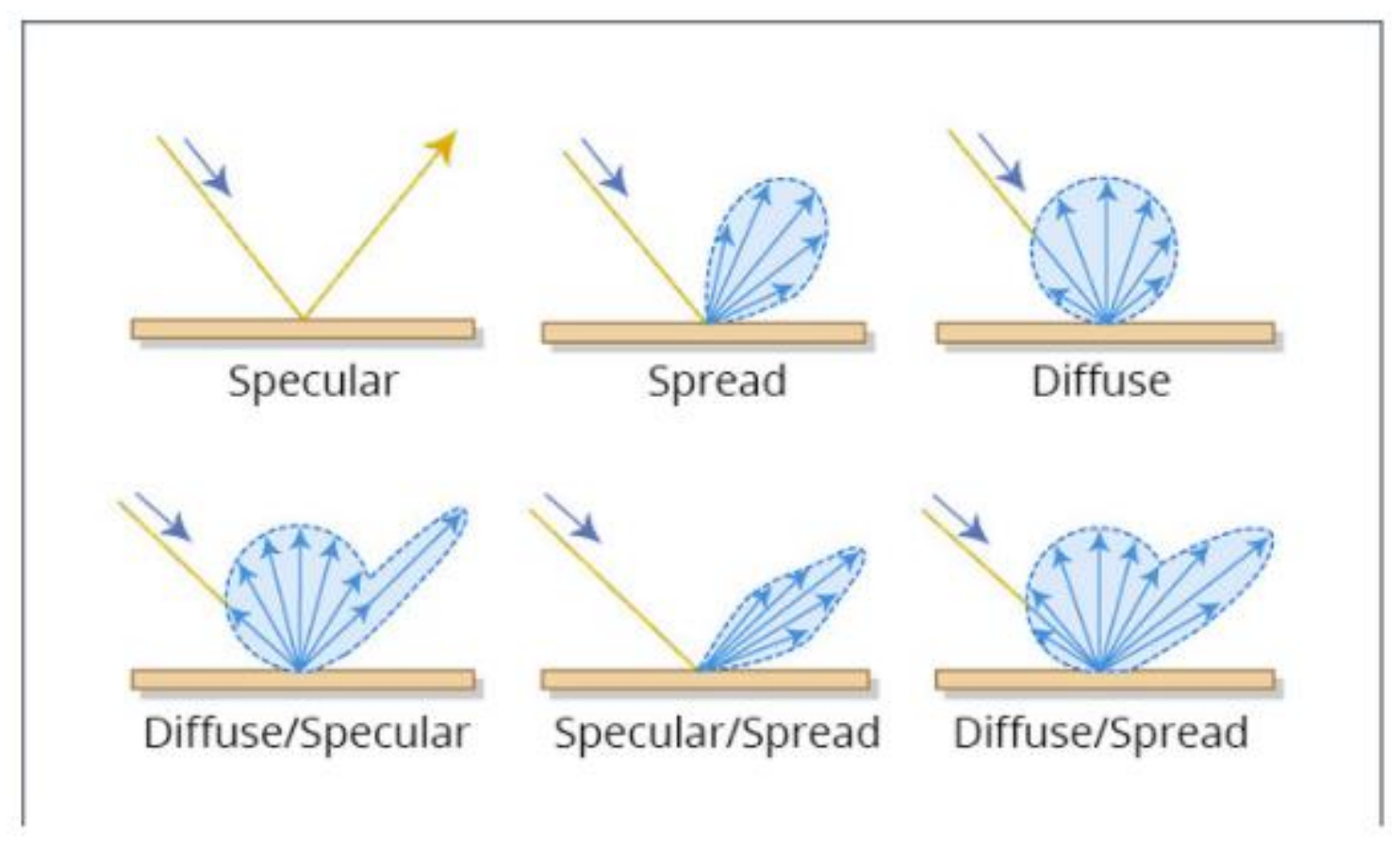

Figure 9: The reflection situation (terabee, 2021)

\subsection{LIDAR System Options}

\section{LIDAR Operational Theory}

- A pulse of light is emitted and the precise time is recorded.

- The reflection of that pulse is detected and the precise time is recorded.

- Using the constant speed of light, the delay can be converted into a "slant range" distance.

- Knowing the position and orientation of the sensor, the $X Y Z$ coordinate of the reflective surface can be calculated. 


\subsubsection{Phase Shift Method for ToF Estimation}

The phase shift method computes the phase difference among emitted and received light by altering the amplitude of the light generated. Aliasing will happen at distances (d) that are a multiple of the speed of light (c) and modulation frequencies (f)

$$
d=c * f / 2 \text {. }
$$

To reduce the impact of this phenomena, other frequencies might be used to increase the sensing range. Direct sampling, heterodyne (because the phase of the signal is not changed at intermediate frequency), and under sampling with I/Q demodulation may all be used to measure phase shift (Chevrier \& Campanella, 2018, P.7/25).

\subsubsection{Pulse ToF Estimation}

The pulse ToF estimate method uses a direct measurement of the ToF between laser light emission and reception (Chevrier \& Campanella, 2018, P.7/25).

\section{Common LiDAR Ranging Arc hitec tures}

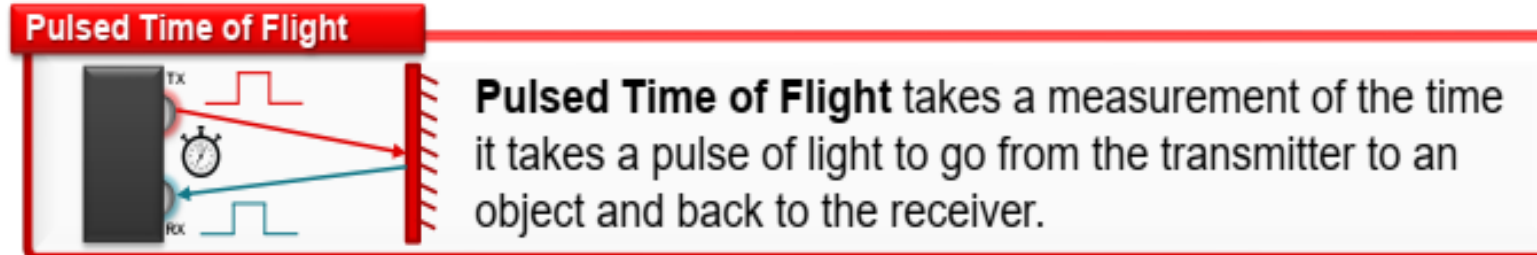

\section{Phase Shift}

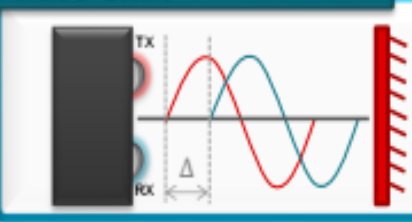

Phase Shift uses a continuous wave and measures the time of flight as a phase shift. The maximum distance is limited by phase wrapping.

Frequency Modulation

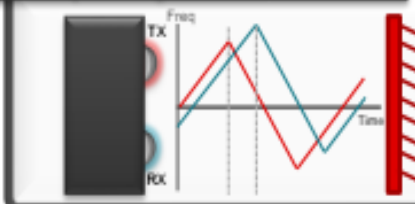

Frequency modulation (FMCW) uses a continuous wave with varying frequency and measures the time of flight as a frequency difference. Distance \& velocity can be measured. 


\subsection{The basic principle of LIDAR}

\section{B as íc LiDAR Princìples}

\section{Pulsed Time of Flight (ToF)}

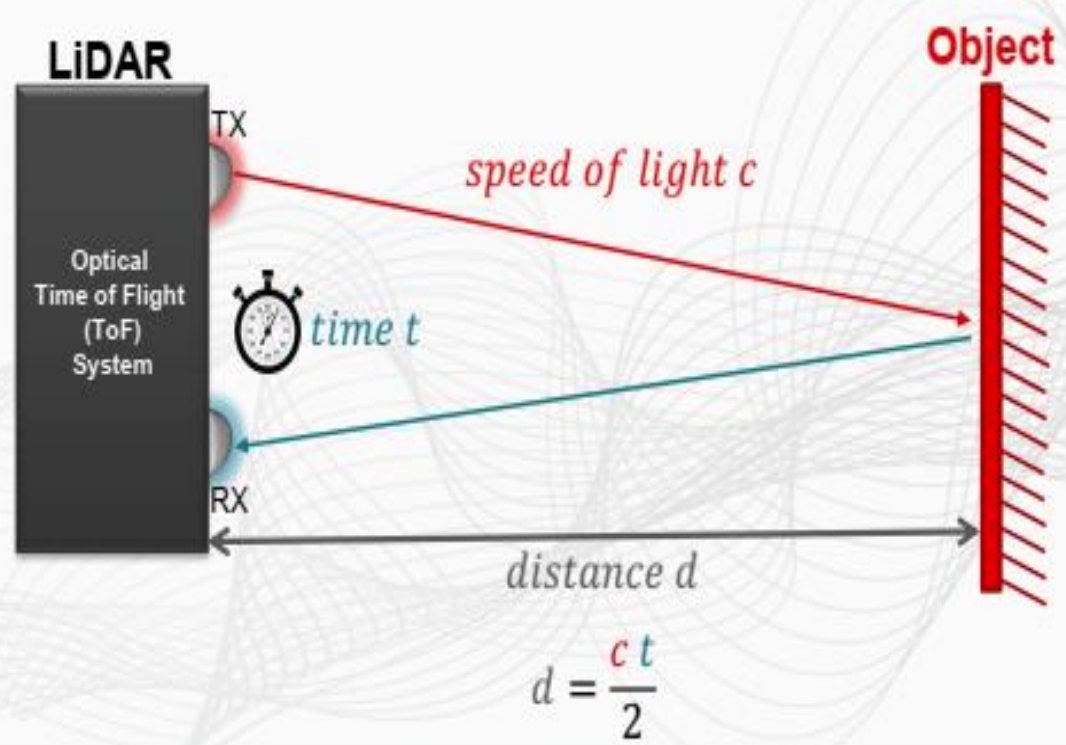

The basic idea behind this technology is to release a light pulse and then receive the returning one in order to determine the time difference between these two pulses. The system will be able to provide the three-dimensional coordinates of the target item based on the time difference, the angle at which the light pulse was shot, and the position of the system itself . As long as laser pulses move at the speed of light, the distance travelled by the pulse may be determined using the following equation:

$$
\text { Distance }=\frac{\text { timetravel } * \text { speed of light }}{2}
$$


And it is multiplied by two since the light goes to the earth and then back to the system (Olsen, 2016, P.11/29).

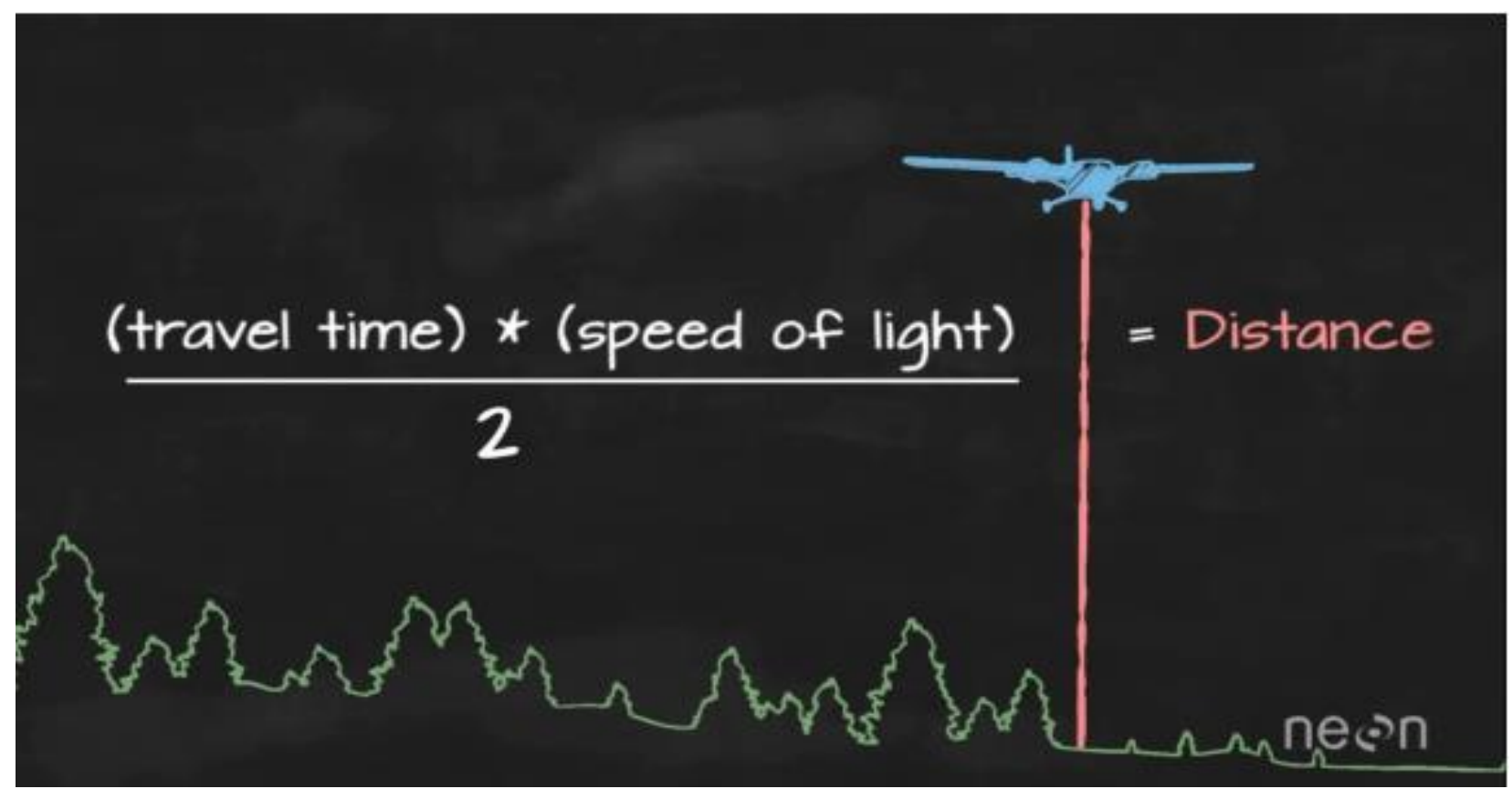

Figure 10: Distance traveled by the laser pulse (Olsen, 2016).

\subsection{Conceptual Discussion of LiDAR}

A waveform generator generates the laser waveform required to determine range or velocity. A laser is often used to give light, though a LiDAR may operate without one. A few LiDAR systems were created prior to the advent of the laser. In principle, any light source might be used, but since its creation, the laser has been the light source of choice for LiDAR. This can be a single laser, a seeded laser, or a master oscillator with one or more laser amplifiers following it. It might also be a laser array. The light must be emitted through a transmit optical aperture. This might be the same or a different aperture than the receive aperture. If it has the same aperture, it is referred to as a monostatic LiDAR, where mono denotes "one aperture." If the transmit and receive apertures differ, the device is referred to as a bistatic LiDAR, which means it has two apertures. We need a pointing technique and a means to find out where to point the laser at the target we want to observe, thus we need a pointing technique and a means to figure out where to point. To reach a destination, light must travel through a medium, generally the atmosphere. 
LiDAR signals may be transferred over space's vacuum. LiDAR may also be employed in water rather than air or space (De Raedt et al., 2009, P.5/28).

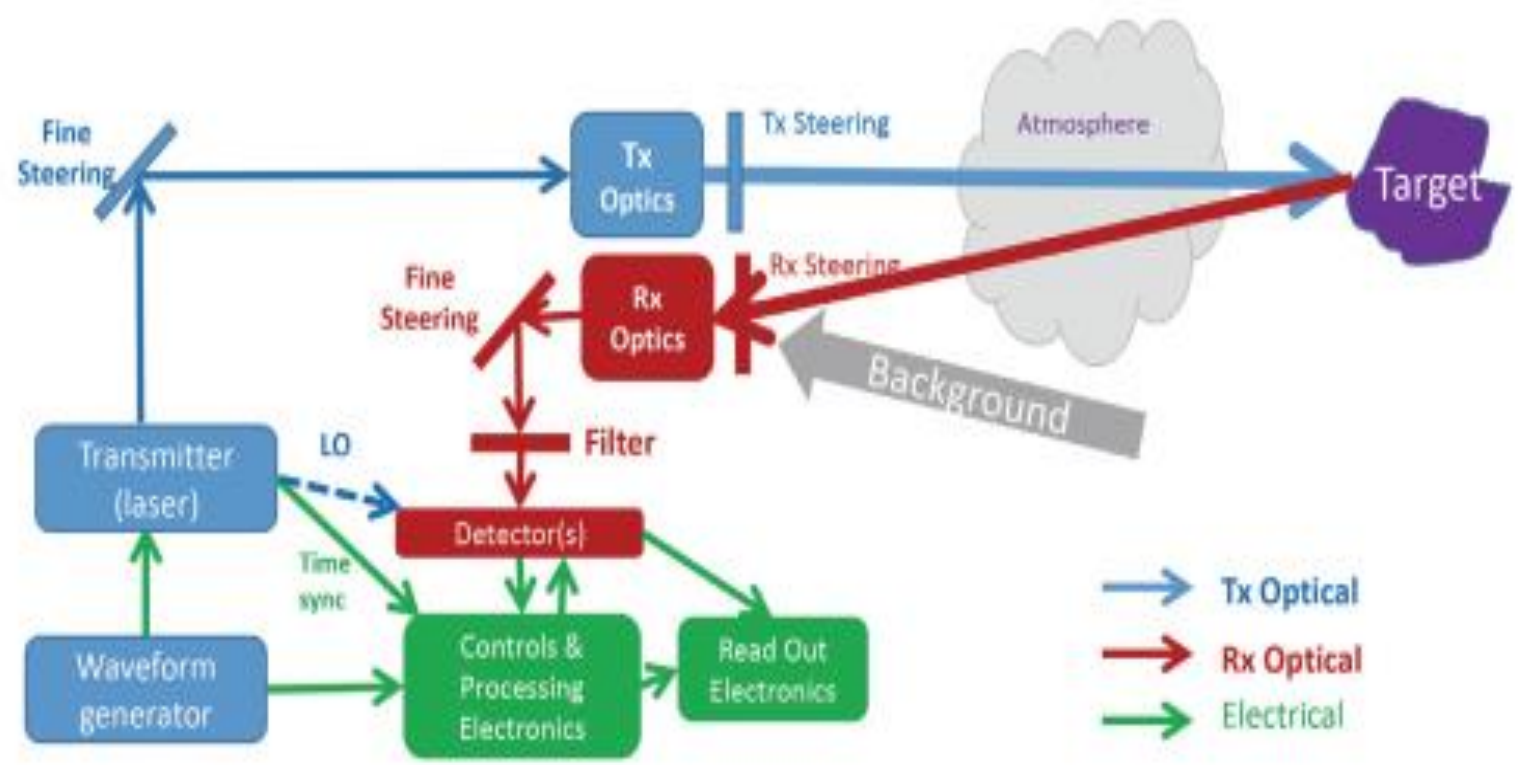

Figure 11: LiDAR Conceptual diagram (De Raedt et al., 2009, P.5/28).

\subsection{LIDAR and Time of Flight}

LIDAR systems and ToF approaches are critical for self-driving cars to have a perfect view of their surroundings, and they're also used in a variety of research projects. The second part of a four-part series on LIDAR systems and ToF methodologies is now available. Light and photons act and manage differently than RF or sound energy, hence LIDAR, like radar and sonar, has a simple principle but a difficult implementation. Nonetheless, LIDAR is successful, and a lightbased system can provide both accurate distance and angular resolution because to the small wavelength of light, among other factors (microcontrollertips, 2019).

The LIDAR system begins with an infrared-emitting laser diode or LED. Direct ToF calculates the distance to an object by sending out short light pulses and calculating the time it takes for each pulse to return to the sensor. Indirect ToF sensors generate a continuous stream of modulated light. A photoreceptor detects any reflections, and the timing and phase of the 
reflected light are used to calculate the distance to the item that created the reflection. Direct ToF pulses are typically several nanoseconds long. Shorter pulses offer higher resolution but lower energy, resulting in a reduced sign/noise ratio (SNR) in the acquired reflection; this is one of numerous tradeoffs (microcontrollertips, 2019).

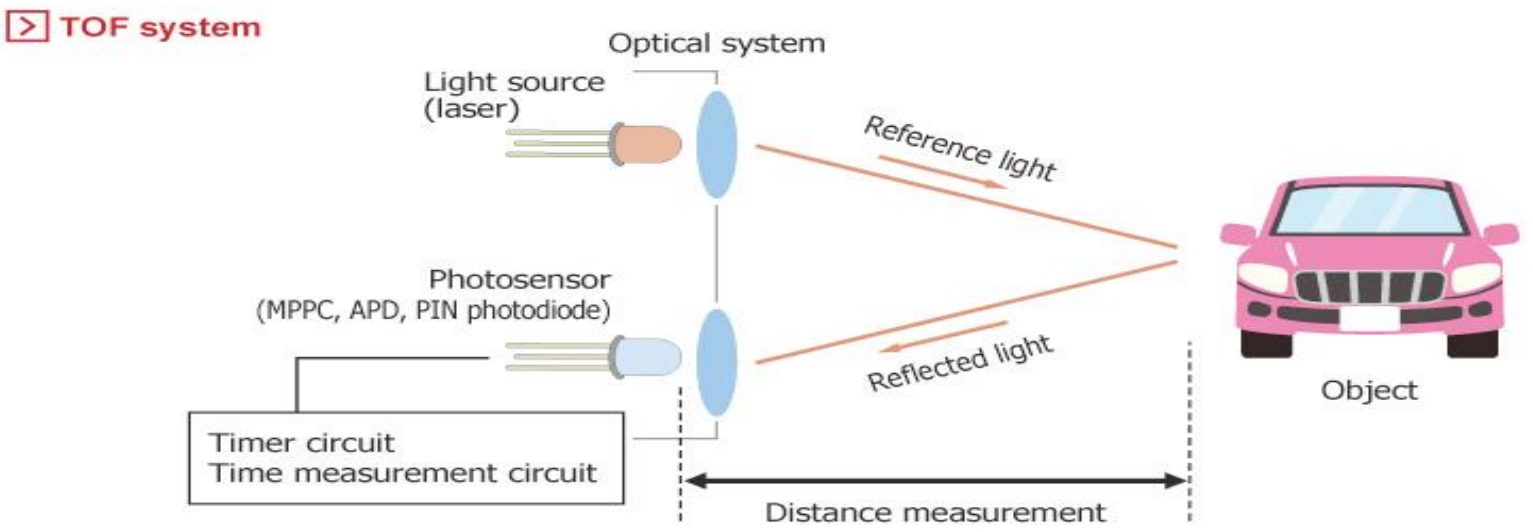

Figure 12: The LIDAR system uses the time of flight a reflection of a light beam (pulsed or continuous) to sense the surroundings (microcontrollertips, 2019).
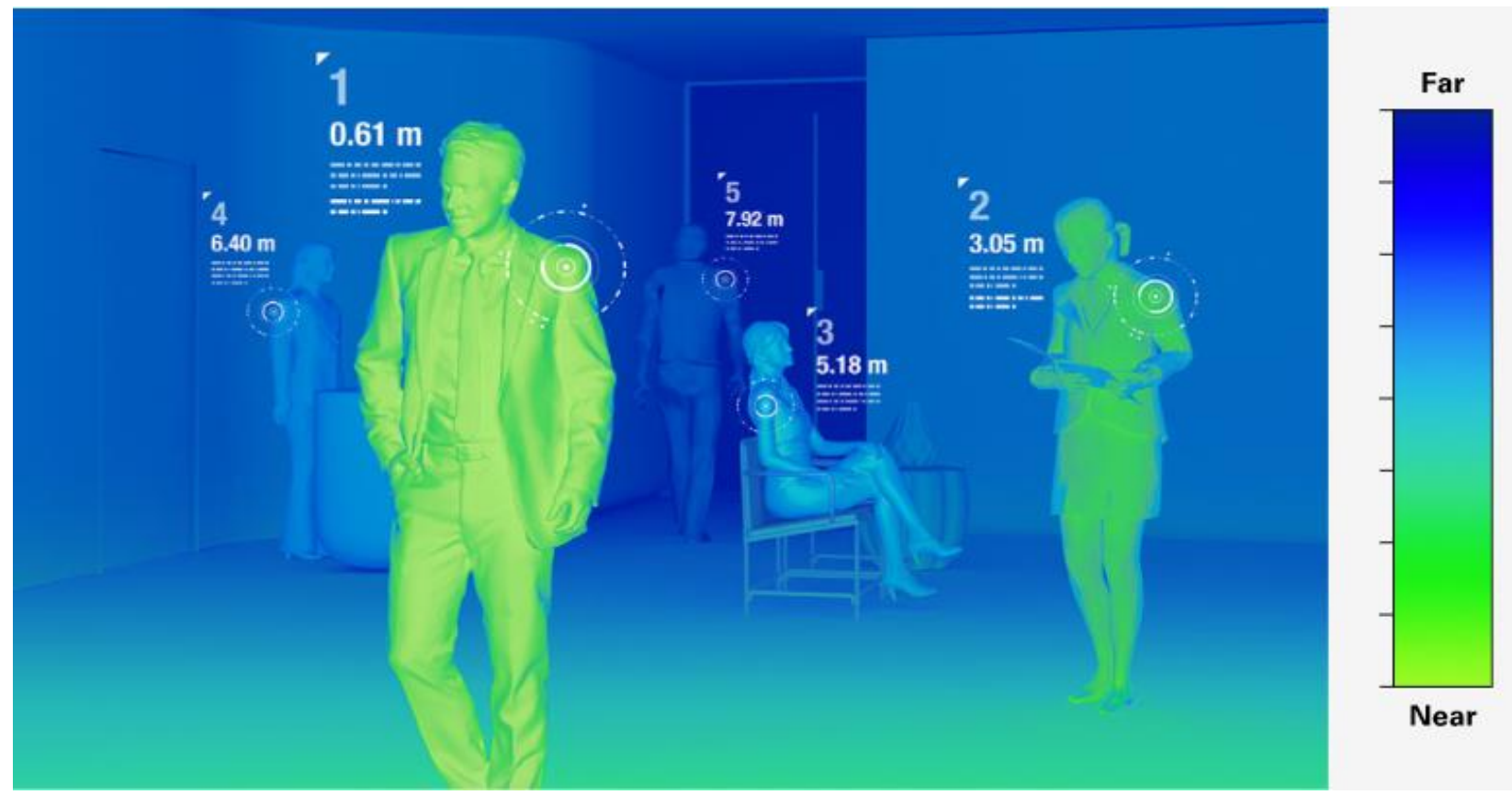

Figure 13: Example image of using time of flight measurement for people classification and range detection (analog.com, 2021). 
ToF enables the capturing of the entire picture at once. There is no need for line-by-line scanning or relative movement between the sensor and the items being observed. ToF is sometimes referred to as LIDAR (light detection and ranging), however this is a flash LIDAR-based approach rather than a scanning LIDAR. ToF may measure the time of flight of a light pulse in two ways: pulsed (using charge coupled device (CCD) technology) and continuous wave (CW) .

The time duration between the emission and reception of a light pulse is measured in pulsed mode, whereas the phase shift between the emitted and received modulated light pulses is determined in CW mode. Both modes of working have advantages and disadvantages . Pulsed mode is more resistant to ambient light and hence more conductive to outdoor applications since this technology typically relies on high intensity light pulses delivered in very brief bursts across a small integration window. CW mode, on the other hand, may be easier to build since the light source does not need to be as short as possible, with sharp rising and falling edges (Brand et al, 2019, P.1/2).

However, if the precision of the requirements grows, higher frequency modulation signals will be required, which may be difficult to obtain. High chip resolutions are achievable because to current pixel sizes, allowing not only distance measurements but also object and gesture recognition. The lengths measured range from a few centimeters $(10 \mathrm{~cm})$ to several meters $(15$ $\mathrm{m})$. Unfortunately, not everything can be recognized to the same degree. The item's condition, reflectivity, and speed all have an effect on the measurement output. Environmental factors like as fog or bright sunlight may affect the measurement result. The latter is aided by the suppression of ambient light (Brand et al., 2019, P.1-2/2). 


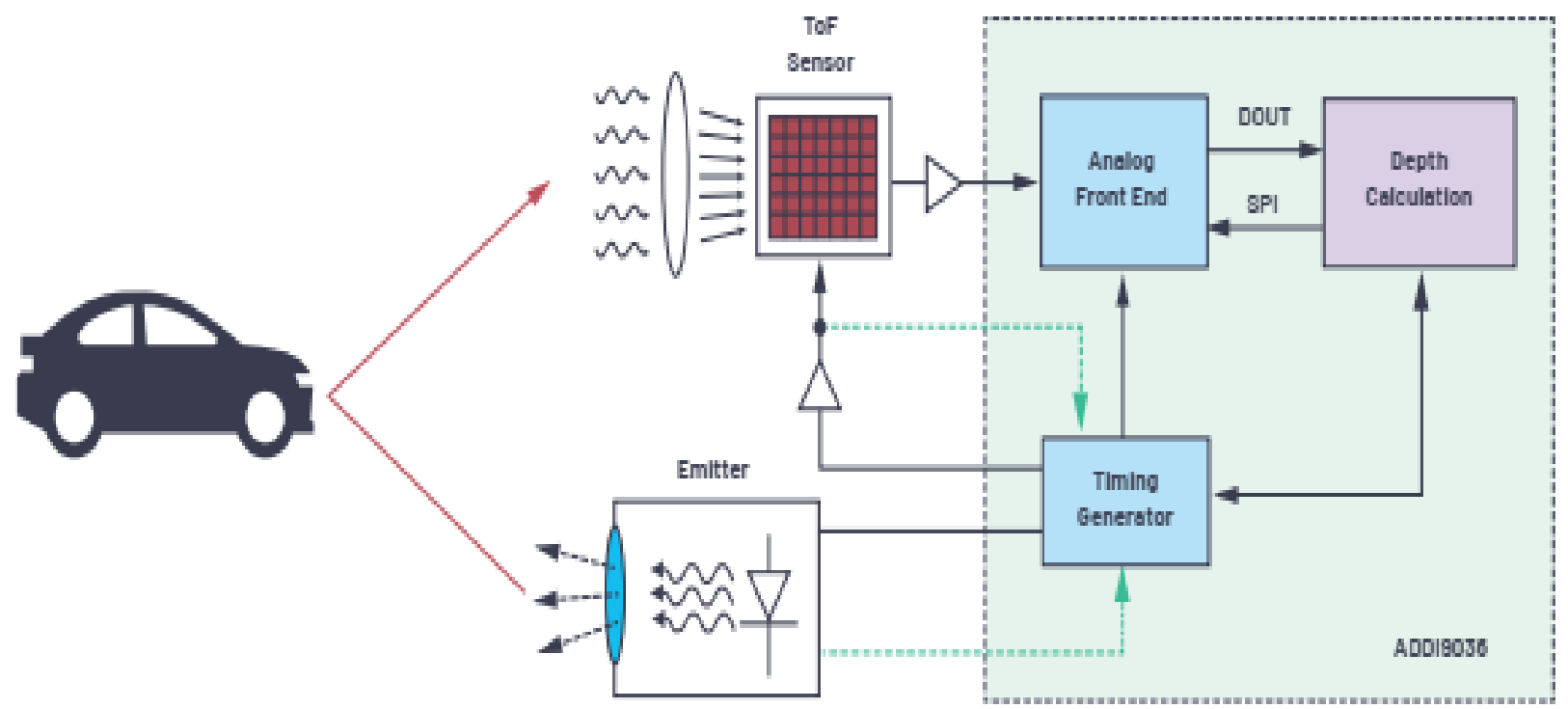

Figure 14: Block diagram of a ToF system (Brand et al., 2019, P.2/2).

\section{Methods and Recommendations}

\subsection{Types of LIDAR Systems}

LIDAR systems are split into three types:

\subsubsection{LIDAR platforms}

There are two basic approaches for gathering data:

* The flown system (Airborne), in which an aero plane is outfitted with a LIDAR system.

* The ground-based stationary (Terrestrial LIDAR), in which the system is anchored to the ground (Olsen, 2016, P.12/29).

\subsubsection{Airborne LiDAR}

To collect data, airborne LiDAR is mounted to a helicopter or drone. When engaged, Airborne LiDAR sends light towards the ground surface, which returns to the sensor instantly after striking the target, providing a precise distance measurement. Topological LiDAR and Bathymetric LiDAR are the two forms of airborne LiDAR (geospatialworld, 2021) 


\section{Principles of Airborne LiDAR - Flight Planning}

- cannot penetrate clouds

- are often flown at night

- overlap of 30 to $50 \%$ in steeper terrain

- multiple passes at different angles in urban areas (to avoid LiDAR "shadow")

- flying elevation typically 200 to 300 meters (higher in urban areas)

- multiple "returns" are received for each laser pulse fired from the scanner

- modern systems are capable of recoding up to 5 returns for each pulse

A LIDAR system that operates from an airborne platform includes several instruments: the laser device; an inertial navigational measurement unit (IMU) that continuously records the aircraft's attitude vectors (orientation); a high-precision airborne global positioning system (GPS) unit that records the aircraft's three-dimensional position; and a computer interface that manages communication (Gatziolis \& Andersen, 2008, P.5/40).

\subsubsection{Terrestrial LiDAR}

Terrestrial LiDAR systems, as opposed to airborne LiDAR systems, are deployed on moving vehicles or tripods on the earth's surface to acquire precise data points. These are commonly used for monitoring roadways, analyzing infrastructure, and even gathering point clouds from the interior and outside of buildings. There are two types of terrestrial LiDAR systems: mobile LiDAR and static LiDAR (geospatialworld, 2021).

Mobile LIDAR is the acquisition of LIDAR point cloud data from a rotating platform. A mobile LIDAR system can be made up of any number of LIDAR sensors installed on a moving object. These gadgets might be used in cars, trains, and sometimes even boats. Mobile LIDAR 
systems, like airborne LIDAR systems, generally include a LIDAR sensor, cameras, GPS (Global Positioning System), and an INS (inertial navigation system). Mobile lidar data may be used to monitor rail network and identify approaching overhead wires, light poles, and road signs near roadways or rail lines (desktop.arcgis, 2019).

Static LIDAR is the collection of LIDAR point clouds from a stationary location. The LIDAR sensor, which is often mounted on a tripod, is a totally portable, laser-based range and imaging technology. These systems can acquire LIDAR point clouds both inside and outside of buildings. Engineering, mining, surveying, and archaeology are all frequent applications for this type of LIDAR (desktop.arcgis, 2019).

\subsubsection{The Origin of Energy}

There are two categories of remote sensing technologies based on the source of energy utilized to identify the target object: passive systems and active systems (Olsen, 2016).

\subsubsection{Passive System}

The passive system identifies items based on the radiations emitted by the object itself, such as near field communication tags.

\subsubsection{Active System}

An active system does not rely on an outside source. It creates energy, directs it to the target item, and then detects the radiation induced by the produced energy.

It appears that relying on a passive system is preferable, as such a system will reduce spent power (Olsen, 2016, P.13/29).

\subsubsection{The Object of Interest}

LIDAR detects whatever the item is; all it takes is for the thing to emit light when it is illuminated. However, in order to detect the seafloor, a LIDAR that can deliver pulses through water is required (Olsen, 2016, P.13/29). 


\subsubsection{Topographic LIDAR}

Topographic LIDAR is most commonly employed in land surveying, urban planning, and landscape ecology (Olsen, 2016, P.13/29).

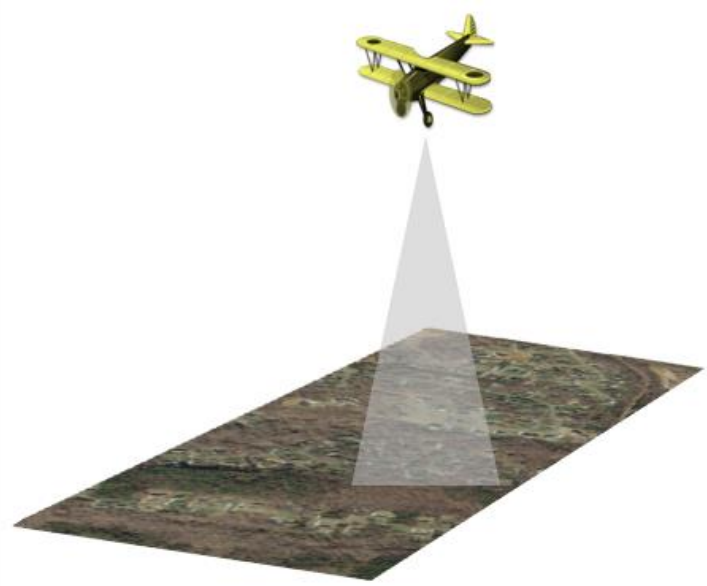

Figure 15: Topographic LIDAR (desktop.arcgis, 2019).

\subsubsection{Bathymetric LIDAR}

Bathymetric LIDAR is a type of airborne water-penetrating technology. Most bathymetric LIDAR systems record both elevation and water depth, resulting in an airborne LIDAR survey of the land-water interface. During a bathymetric LIDAR scan, the infrared light (standard laser system) is reflected back to the aircraft from the land and water surface, while the additional green laser enters into the water column (Olsen, 2016, P.13/29).

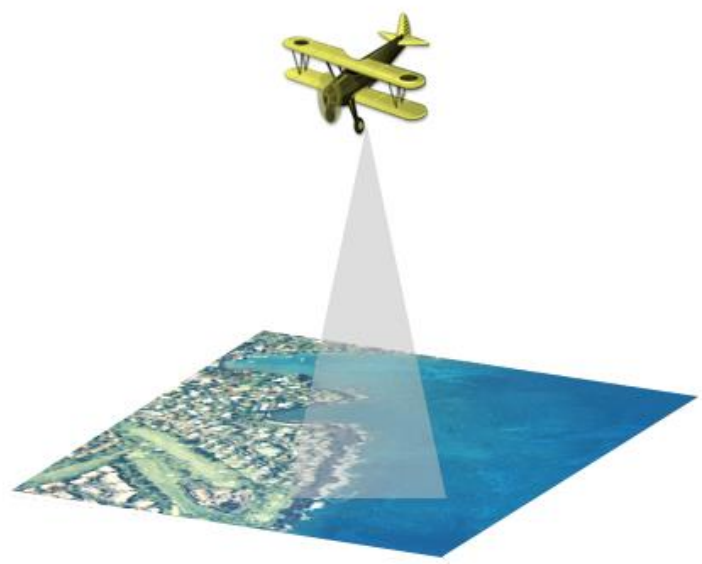

Figure 16: Bathymetric LIDAR (desktop.arcgis, 2019). 


\subsection{How the LIDAR identifies the different objects}

Qualities that enable LIDAR to provide high-quality information to users

The LIDAR system captures the following data throughout the surveying process:

I) The angle at which the laser beam was shot.

II) The time gap between the emitted and returning pulses.

III) The Intensity, which is a highly essential metric, relates to the magnitude of the return signal.

Materials with variable spectral reflectance qualities result in varying backscattering laser intensities, in theory. As a result, the LIDAR intensity may be utilized to identify and detect various materials in scans of natural or urban areas (Olsen, 2016, P.15/29).

\subsubsection{Multiple return feature}

Because the device can catch up to five returns per pulse, the multiple return feature allows LIDAR technology to improve its capacity to look at three-dimensional structures. This is due to the fact that the pulse of light does not merely reflect and return from the top of the tree, but it also penetrates everything (Olsen, 2016, P.15/29). 


\subsection{Time-of-flight (TOF) principle technology comparison}

\section{TECHNOLOGY COMPARISON}

Distance sensor for robotics

\begin{tabular}{l} 
Ultrasound Infrared triangulation \\
$\begin{array}{l}\text { High reading } \\
\text { frequency }\end{array}$ \\
\hline Long range \\
\hline Minimal weight \\
\hline Small form factor \\
\hline Eye safety
\end{tabular}

Figure 17: Technology Comparison (terabee, 2021).

\subsection{Advantages and Disadvantages}

Using the rotating micrometer technique, the geometric details of the objects are efficiently retrieved from the measurements provided by the 3-D occupancy grid. When radar data is combined with LIDAR measurements, it provides information about the dynamic properties of the obstacle, such as velocity and location of the obstacle for the sensor location, which helps the 
vehicle or driver decide what action to take to ensure safety. The sole worry is the computational cost of implementing this data processing methodology.

\section{Advantages of LiDAR}

- all data geo-referenced from inception

- high level of accuracy

- ability to cover large areas quickly

- quicker turnaround, less labor intensive, and lower costs than photogrammetric methods

- can collect data in steep terrain and shadows

- can produce DEM and DSM

\section{Disadvantages of LiDAR}

- inability to penetrate very dense canopy leads to elevation model errors

- very large datasets that are difficult to interpret and process

- no international protocols

- cost

- \$200 - \$300 / sq mile - 3 meters resolution

- \$350 - \$450 / sq mile - 1 meter resolution 


\subsection{Applications}

The primary concept is to measure the time difference between the emitted and returned pulses, record the laser angle, and then find the target.

The primary purpose of this technology at the outset was to detect the ground of the target region and determine its elevation. The initial application of LIDAR with pulsed ToF data was to create attractive high resolution elevation maps to find holes, picks, hills, and even streams and roads in previously unknown locations. Following are the applications of LIDAR with P-ToF,

* Architectural Surveying Tools

* LIDAR for Automotive Scanning

* Drones

* Gas Detection

* Laser Range Finder (LIDAR)

* Laser Scanners for Safety

* Imaging of the retina

* Automation and robotics (Texas Instruments, 2017, P.1/29)

Also For a long time, trees were thought to be a source of noise in the survey process, and people worked hard to eliminate as much noise as possible in order to get high resolution elevation data. Later, they found that the noise they were attempting to eliminate included highly vital information that may be highly valuable!

Since then, LIDAR has been used to determine the height of trees and structures, to determine the best time for harvesting, and to identify natural and urban cover surfaces. Furthermore, it is utilized as a complement to data detecting natural environments:

- Differentiation between rock and soil layers.

* Detection of changes in snow cover.

* Wetland hydrology and flood modelling

* The lava flows are ageing.

* Mapping of coastal land cover. 
* Classification of trees, identification of snags (Olsen, 2016, P.22/29)

\subsubsection{Oceanography}

LiDAR technology is used by authorities to determine the precise depth of the ocean's surface in order to find any object in the event of a maritime mishap or for research reasons. Aside from finding items, LiDAR is also used to calculate phytoplankton fluorescence and biomass at the ocean's surface, which is otherwise difficult (geospatialworld, 2021).

\subsubsection{Digital Elevation or Terrain Model}

Terrain elevations are extremely important in the construction of roadways, huge structures, and bridges. Because LiDAR technology contains $\mathrm{x}, \mathrm{y}$, and z coordinates, it is relatively simple to create $3 \mathrm{D}$ representations of elevations so that interested parties may draw appropriate conclusions more simply (geospatialworld, 2021).

\subsubsection{Agriculture \& Archaeology}

Typical agricultural applications of LiDAR technology include production rate analysis, crop reconnaissance, and seed dispersions. It is also utilized for campaign planning, mapping under the forest canopy, and other purposes (geospatialworld, 2021).

\section{Conclusion}

With the advancement of LIDAR systems, they are becoming increasingly helpful in a variety of applications, and the entire world will rely on various LIDAR systems to discover, gather, and update data from various areas. For many IoT-enabled applications, ToF is a diverse and precise distance measurement solution. New sensor generations expand a product's capability, improve dependability, and give a competitive advantage. Sensors that were initially designed for a certain market are increasingly finding homes in unexpected places.

Accuracy, resolution, and linearity are all important factors to consider when measuring 3D using LIDAR. One of the most significant aspects of LIDAR design is the optical system. According to the experimental results, appropriate optical systems were examined in this study based on the required detection range and distance resolution. Also because of its great precision and 
resolution, LIDAR outperforms pre-existing RADAR and SONAR technology. 3D mapping is extremely useful in industries such as rescue, agriculture, and wind farm optimization. 3D mapping with LIDAR is incredibly exact, reliable, and cost-effective. The LIDAR is connected to a pan tilt servo motor kit, which creates a 3D map of the front hemisphere. As a result, this study discusses various 3D optical measurements utilizing LIDAR with pulsed time of flight, as well as the use of LIDAR in many sectors. 


\section{References}

analog.com. (2021). 3D IMAGING WITH ADI TIME OF FLIGHT TECHNOLOGY. https://www.analog.com/en/applications/technology/3d-time-of-flight.html\#

Brand, T., Field, S., \& Engineer, A. (n.d.). Time of Flight System for Distance Measurement and Object Detection. 1-2.

Chevrier, M., \& Campanella, G. (2018). LIDAR Pulsed Time of Flight Reference Design. January 2018, 1-25.

De Raedt, W., Carchon, G., Tilmans, H. A. C., \& Beyne, E. (2009). RF technologies and systems. More than Moore: Creating High Value Micro/Nanoelectronics Systems, 63-84. https://doi.org/10.1007/978-0-387-75593-9_3

desktop.arcgis. (2019). What is lidar data? https://desktop.arcgis.com/en/arcmap/10.3/managedata/las-dataset/what-is-lidar-data-.htm

Fang, Z., Zhao, S., Wen, S., \& Zhang, Y. (2018). A Real-Time 3D Perception and Reconstruction System Based on a 2D Laser Scanner. Journal of Sensors, 2018. https://doi.org/10.1155/2018/2937694

Gatziolis, D., \& Andersen, H. E. (2008). A guide to LIDAR data acquisition and processing for the forests of the pacific northwest. USDA Forest Service - General Technical Report PNWGTR, 768, 1-32. https://doi.org/10.2737/PNW-GTR-768

geospatialworld. (2021). What is LiDAR technology and how does it work? Bhupendra Sharma. https://www.geospatialworld.net/blogs/what-is-lidar-technology-and-how-does-it-work/

Hildebrandt, M., Kerdels, J., Albiez, J., \& Kirchner, F. (2008). A practical underwater 3DLaserscanner. Oceans 2008, May 2014. https://doi.org/10.1109/OCEANS.2008.5151964

Maric, J., \& Siedersbeck, A. (2018). Time-of-flight (ToF) measurement using pulse lasers. 1-22. https://dammedia.osram.info/media/resource/hires/osram-dam-2496586/Time-of-flight (ToF) measurement using pulse lasers.pdf

microcontrollertips. (2019). LIDAR and Time of Flight, Part 2: Operation. https://www.microcontrollertips.com/lidar-and-time-of-flight-part-2-operation/

Myllylä, R., Marszalec, J., Kostamovaara, J., Mäntyniemi, A., \& Ulbrich, G.-J. (1998). Imaging distance measurements using TOF lidar. Journal of Optics, 29(3), 188-193. https://doi.org/10.1088/0150-536x/29/3/016

Olsen, R. C. (2016). Light Detection and Ranging. Remote Sensing from Air and Space, Second Edition, July. https://doi.org/10.1117/3.2234477.ch11

Sarbolandi, H., Plack, M., \& Kolb, A. (2018). Pulse based time-of-flight range sensing. Sensors (Switzerland), 18(6). https://doi.org/10.3390/s18061679

terabee. (2021). A Brief Introduction to Time-of-Flight Sensing: Part 1 - The Basics. 
https://www.terabee.com/a-brief-introduction-to-time-of-flight-sensing-part-1-the-basics/

Texas Instruments. (2017). LIDAR-Pulsed Time-of-Flight Reference Design Using High-Speed Data Converters. November 2016, 1-29. www.ti.com

Tontini, A., Gasparini, L., \& Perenzoni, M. (2020). Numerical model of spad-based direct timeof-flight flash lidar CMOS image sensors. Sensors (Switzerland), 20(18), 1-19.

https://doi.org/10.3390/s20185203 